\title{
Highly flexible strain sensor from tissue paper for
}

\section{wearable electronics}

Yuanqing $\mathrm{Li}^{1^{*}}$, Yarjan Abdul Samad ${ }^{2}$, Tarek Taha ${ }^{3}$, Guowei Cai ${ }^{3}$, Shao-Yun Fu ${ }^{1}$, Kin Liao ${ }^{2 *}$

1. College of Aerospace Engineering, Chongqing University, No. 174 Shazhengjie Road, Chongqing 400044, People's Republic of China

2. Department of Mechanical Engineering, Khalifa University of Science, Technology, \& Research, Abu Dhabi 127788, United Arab Emirates

3. Robotics Institute, Khalifa University of Science, Technology \& Research, Abu Dhabi 127788, United Arab Emirates

*Address correspondence to yqli@cqu.edu.cn; kin.liao@kustar.ac.ae

\section{Supporting information, 3 pages and 4 figures}

Figure S1. EDX spectrum of carbon paper prepared.

Figure S2. The stress of CP/PDMS sensor with cyclic tensile strain applied.

Figure S3. Response of CP/PDMS with cyclic tensile, compressive, and bending strain applied.

Figure S4. Schematic of the dimension and size of the CS/PDMS strain sensor. 


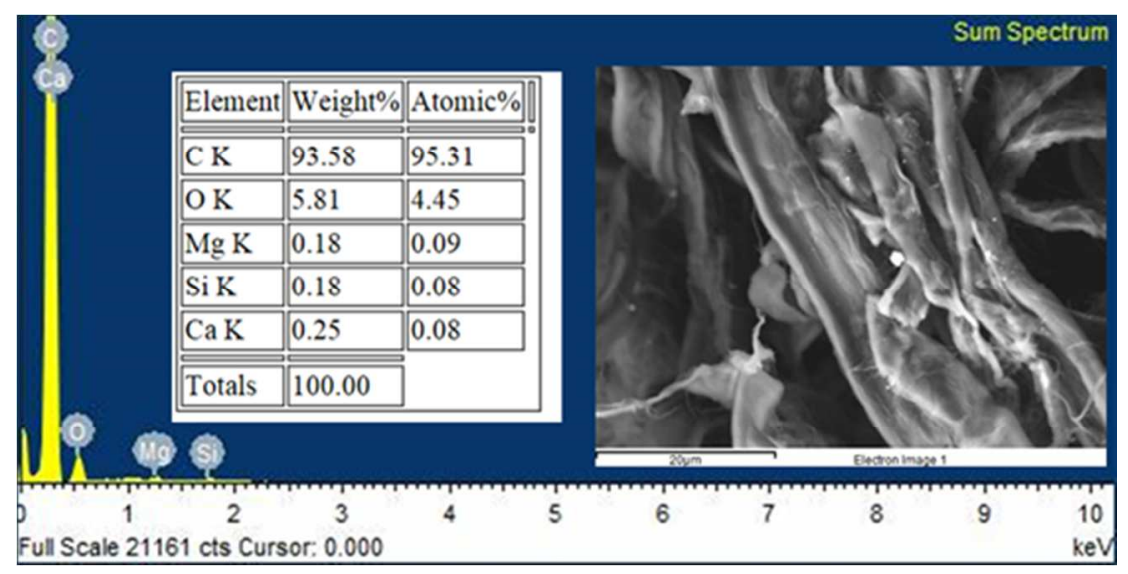

Figure S1. EDX spectrum of carbon paper prepared.

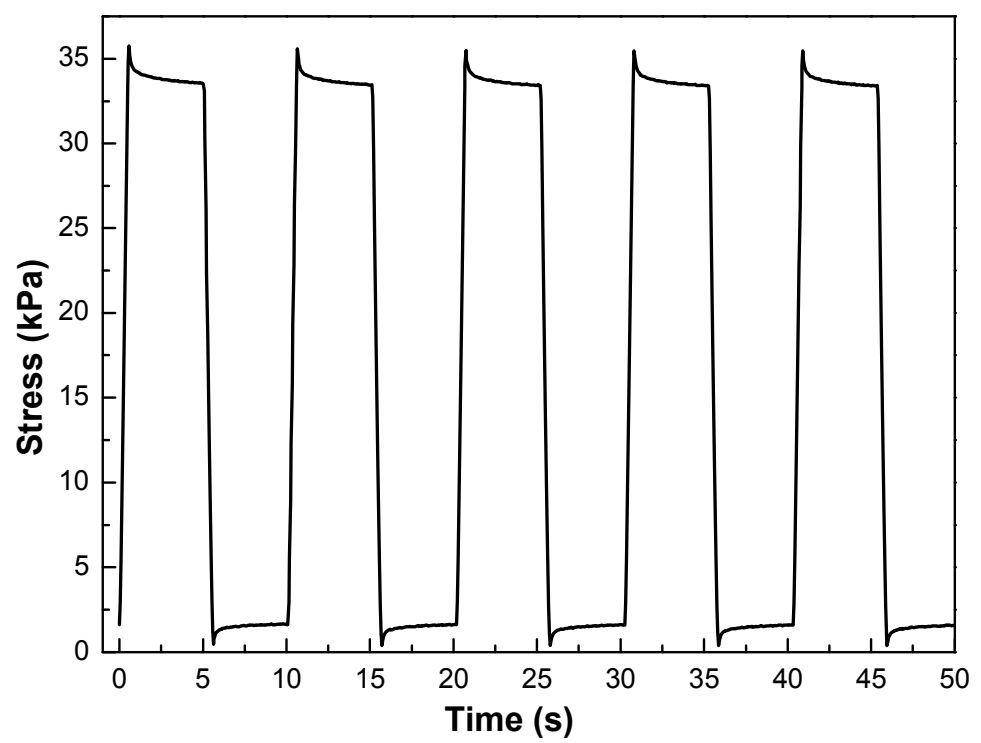

Figure S2. The stress of CP/PDMS sensor with cyclic tensile strain applied. 


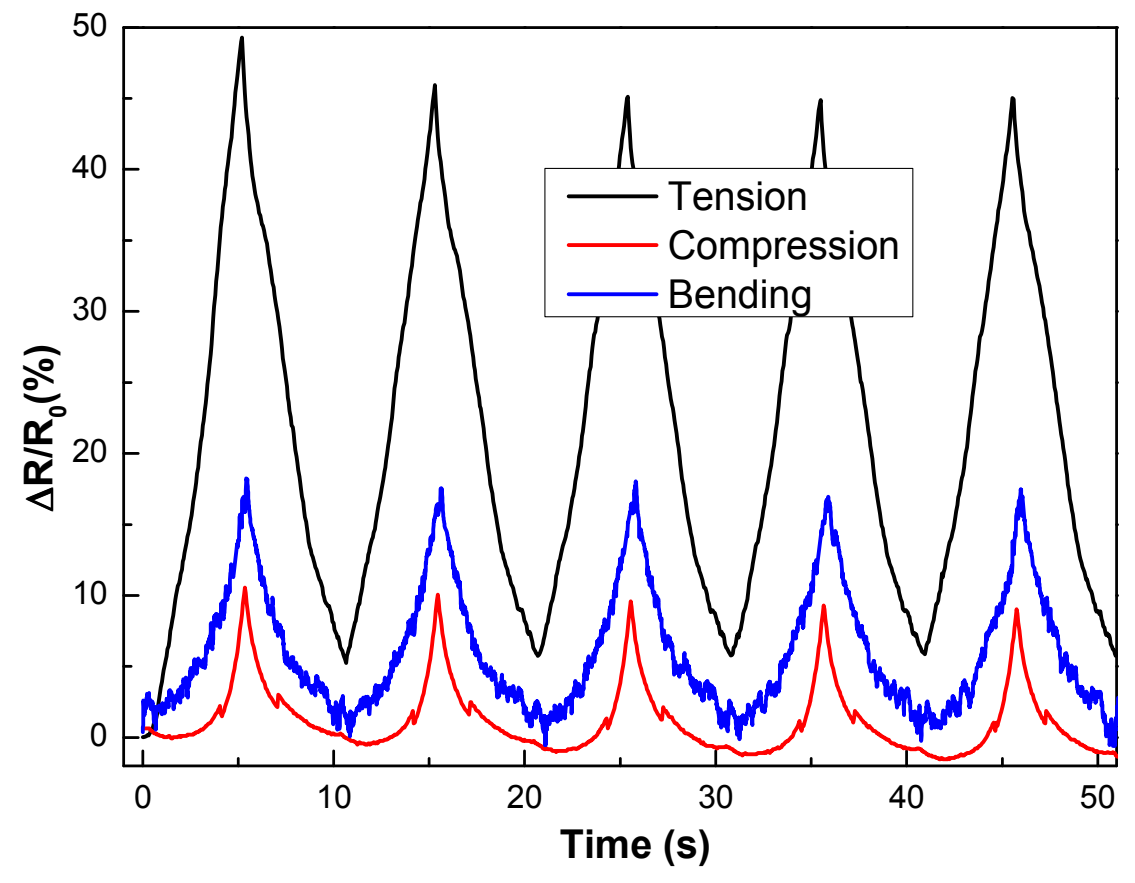

Figure S3. Response of CP/PDMS with cyclic tensile, compressive, and bending strain applied.

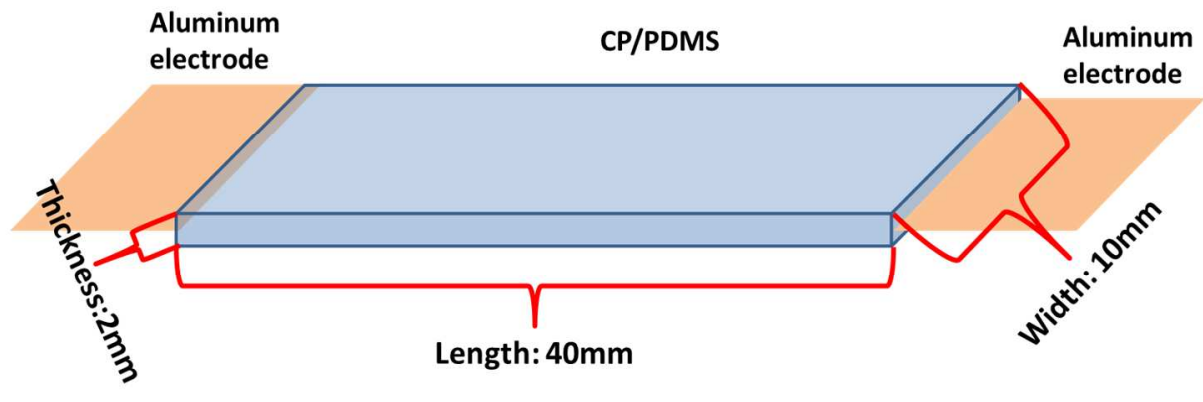

Figure S4. Schematic of the dimension and size of the CS/PDMS strain sensor. 